\title{
Linear Programming Problem with Interval Type 2 Fuzzy Coefficients and an Interpretation for Its Constraints
}

\author{
A. Srinivasan and G. Geetharamani \\ Department of Mathematics, Anna University, Bharathidasan Institute of Technology (BIT) Campus, Tiruchirappalli, \\ Tamilnadu 620 024, India \\ Correspondence should be addressed to A. Srinivasan; yeacheenu4phd@gmail.com
}

Received 16 August 2015; Accepted 3 November 2015

Academic Editor: Frank Werner

Copyright ( 2016 A. Srinivasan and G. Geetharamani. This is an open access article distributed under the Creative Commons Attribution License, which permits unrestricted use, distribution, and reproduction in any medium, provided the original work is properly cited.

\begin{abstract}
Interval type 2 fuzzy numbers are a special kind of type 2 fuzzy numbers. These numbers can be described by triangular and trapezoidal shapes. In this paper, first, perfectly normal interval type 2 trapezoidal fuzzy numbers with their left-hand and righthand spreads and their core have been introduced, which are normal and convex; then a new type of fuzzy arithmetic operations for perfectly normal interval type 2 trapezoidal fuzzy numbers has been proposed based on the extension principle of normal type 1 trapezoidal fuzzy numbers. Moreover, in this proposal, linear programming problems with resources and technology coefficients are perfectly normal interval type 2 fuzzy numbers. To solve this kind of fuzzy linear programming problems, a method based on the degree of satisfaction (or possibility degree) of the constraints has been introduced. In this method the fulfillment of the constraints can be measured with the help of ranking method of fuzzy numbers. Optimal solution is obtained at different degree of satisfaction by using Barnes algorithm with the help of MATLAB. Finally, the optimal solution procedure is illustrated with numerical example.
\end{abstract}

\section{Introduction}

Linear programming and its applications are used in many fields of operations research. It is concerned with the optimization of a linear function while satisfying a set of linear equality and/or inequality constraints or restriction. In real world situation, a linear programming model involves a lot of parameters whose values are assigned by experts. However, both experts and decision makers often do not precisely know the value of those parameters. Therefore, it is useful to consider the knowledge of experts about the parameters as fuzzy data. To make this possible Zadeh [1] introduced fuzzy set theory. The theory proposes a mathematical technique for dealing with imprecise concepts and problems that have many possible solutions. The concept of fuzzy mathematical programming on general level was first proposed by Tanaka et al. [2] in the framework of the fuzzy decision-making in fuzzy environment given by Bellman and Zadeh [3].

The concept of fuzzy liner programming was first formulated by Zimmermann [4]. After this motivation and inspiration several authors such as Deng et al. discussed the fact that the fuzzy bilevel linear programming with multiple followers model solved this complex problem by using the fuzzy structured element method [5]. A new interior point method has been presented to solve fuzzy number linear programming problems using linear ranking function by Zhong et al. [6]. A fully fuzzy linear programming problem with fuzzy equality constraints has been discussed and solved using a compromise programming approach by Cheng et al. [7]. An intuitionistic fuzzy chance constraints model (CCM) based on possibility and necessity measures has been developed and proposed a new method for solving an intuitionistic fuzzy CCM using chance operators by Chakraborty et al. [8]. All the above authors and many others have considered various types of fuzzy linear programming problems and proposed several approaches for solving these problems. Among them, Le and Gogne introduced a class of linear programming problems based on the possibility and necessity relation [9]. Figueroa-García and Hernández proposed an extension of the fuzzy linear programming method which 
was proposed by Zimmermann to an interval type 2 fuzzy linear programming problem with linear membership function [10]. In another work García presented a general model for linear programming where its technological coefficients are assumed as interval type 2 fuzzy sets and it is solved through an $\alpha$-cuts approach [11]. Furthermore García [12] presented a general model to handle uncertainty to right-hand side parameters of a linear programming model by interval type 2 fuzzy sets and trapezoidal membership functions to optimize by using classical algorithms. Chen and Lee [13] proposed the definition of possibility degree of trapezoidal interval type 2 fuzzy numbers and some arithmetic operations. Hu et al. [14] proposed a new approach based on possibility degree to solve multicriteria decision-making problems in which the criteria value takes the form of interval type 2 fuzzy number.

Based on these literatures' survey, with our best knowledge, however, none of them introduced fuzzy linear programming model with both the right-hand side (resources) and the technological coefficients being perfectly normal interval type 2 fuzzy numbers based on possibility. In this paper, first, we have presented a method to do Perfectly normal Interval Type 2 Fuzzy Number (PnIT2FN) arithmetic operation using well-known arithmetical operations on type 1 fuzzy numbers. Further the relation of possibility for PnIT2FN as well as its property are discussed. And a fuzzy linear programming model with both the righthand side (resources) and the technological coefficients being PnIT2FNs has been proposed. By using Zadeh's extension principle, a pair of upper and lower linear problems are developed to calculate the optimal solution of upper and lower bounds of linear problems measure at different possibility level $\alpha$.

The rest of this paper is organized as follows. Firstly Section 2 deals with some preliminary definitions which are given. In Section 3, the definitions of interval type 2 fuzzy sets and trapezoidal interval type 2 fuzzy number have been recalled. In Section 4, the new representation for PnIT2TrFN, its properties, and some arithmetic operations of PnIT2TrFN based on type 1 fuzzy number are presented. The possibility degree of PnIT2TrFN is discussed in Section 5. The fuzzy linear programming problem with the right-hand side and the technological coefficients model is presented in Section 6; Section 7 has numerical illustrations of proposed fuzzy linear programming model with PnIT2TrFN; finally Section 8 has the conclusion of the work.

\section{Preliminaries}

Definition 1 (see [15]). Let $X$ be a nonempty set. A fuzzy set $\widetilde{A}$ in $X$ is characterized by its membership function $\mu_{\widetilde{A}}: X \rightarrow$ $[0,1]$ and $\mu_{\widetilde{A}}(x)$ is interpreted as the degree of membership of element $x$ in fuzzy set $\widetilde{A}$ for each $x \in X$. It is clear that $\widetilde{A}$ is completely determined by the set of tuples

$$
\widetilde{A}=\left\{\left(x, \mu_{\widetilde{A}}(x)\right) \mid x \in X\right\} .
$$

Definition 2 (see [15]). Let $\widetilde{A}$ be a fuzzy subset of $X$ : the support of $\widetilde{A}$, denoted by $\operatorname{Supp}(\widetilde{A})$, is the crisp subset of
$X$ whose elements all have nonzero membership grades in $\widetilde{A}$ :

$$
\operatorname{Supp}(\widetilde{A})=\left\{x \in X \mid \mu_{\widetilde{A}}(x)>0\right\}
$$

Definition 3 (see [15]). A fuzzy subset $\widetilde{A}$ of a classical set $X$ is called normal if there exists $x \in X$ such that $\mu_{\widetilde{A}}(x)=1$. Otherwise $\widetilde{A}$ is subnormal.

Definition 4. An $\alpha$-level set (or $\alpha$-cut) of a fuzzy set $\widetilde{A}$ of $X$ is a nonfuzzy set denoted by $\widetilde{A}_{\alpha}$ and defined by

$$
\widetilde{A}_{\alpha}= \begin{cases}\left\{x \in X \mid \mu_{\widetilde{A}}(x) \geq \alpha\right\}, & \text { if } \alpha>0, \\ \operatorname{cl}(\operatorname{Supp} \widetilde{A}), & \text { if } \alpha=0\end{cases}
$$

where $\operatorname{cl}(\operatorname{Supp} \widetilde{A})$ denotes the closure of the support of $\widetilde{A}$.

Definition 5 (see [15]). A fuzzy set $\widetilde{A}$ of $X$ is called convex if $\widetilde{A}_{\alpha}$ is a convex subset of $X$ for all $\alpha \in[0,1]$.

Definition 6 (see $[15,16])$. A fuzzy number $\widetilde{A}$ is a fuzzy set of the real lines with a normal (fuzzy), convex, and continuous membership function of bounded support. Alternatively, the fuzzy subset $\widetilde{A}$ of $\mathbb{R}$ is called a fuzzy number if the following conditions are satisfied:

(i) $\widetilde{A}$ is normal; that is, there exists $x \in \mathbb{R}$ such that $\mu_{\widetilde{A}}(x)=1$;

(ii) the membership function $\mu_{\widetilde{A}}(x)$ is quasiconcave; that is, $\mu_{\widetilde{A}}\left(\lambda x_{1}+(1-\lambda) x_{2}\right) \geq \min \left\{\mu_{\widetilde{A}}\left(x_{1}\right), \mu_{\widetilde{A}}\left(x_{2}\right)\right\}$ for all $\alpha \in[0,1]$

(iii) the membership function $\mu_{\widetilde{A}}(x)$ is upper semicontinuous; that is, $\left\{x \in \mathbb{R}: \mu_{\widetilde{A}}(x) \geq \alpha\right\}$ is a closed subset of $\mathbb{R}$ for all $\alpha \in[0,1]$;

(iv) the 0 -level set $\widetilde{A}_{\alpha=0}$ is compact (closed and bounded in $\mathbb{R})$.

We denote by $F(\mathbb{R})$ the set of all fuzzy numbers. If $\widetilde{A}$ is a fuzzy number, then from Zadeh [1] $\alpha$-level set $\widetilde{A}_{\alpha}$ is a convex set from condition (ii). Combining this fact with condition (iii), the $\alpha$-level set $\widetilde{A}_{\alpha}$ is a compact and convex set for all $\alpha \in[0,1]$ (since $\widetilde{A}_{\alpha=0}$ is bounded, it says that $\widetilde{A}_{\alpha} \subseteq \widetilde{A}_{\alpha=0}$ is also bounded for all $\alpha \in(0,1])$. Therefore, we can write $\widetilde{A}_{\alpha}=\left[a_{\alpha}^{L}, a_{\alpha}^{U}\right]$.

Definition 7 (see [9]). The trapezoidal fuzzy number is fully determined by quadruples $\left(a^{L}, a^{U}, \alpha, \beta\right)$ of crisp numbers such that $a^{L} \leq a^{U}, \alpha \geq 0$, and $\beta \geq 0$, whose membership function can be denoted by

$$
\mu_{\widetilde{A}}(x)= \begin{cases}\frac{\left(x-a^{L}+\alpha\right)}{\alpha}, & a^{L}-\alpha \leq x \leq a^{L}, \\ 1, & a^{L} \leq x \leq a^{U}, \\ -\frac{\left(x-a^{U}-\beta\right)}{\beta}, & a^{U} \leq x \leq a^{U}+\beta, \\ 0, & \text { otherwise. }\end{cases}
$$


When $a^{L}=a^{U}$, the trapezoidal fuzzy number becomes a triangular fuzzy number. If $\alpha=\beta$, the trapezoidal fuzzy number becomes a symmetrical trapezoidal fuzzy number. $\left[a^{L}, a^{U}\right]$ is the core of $\widetilde{A}$, and $\alpha \geq 0, \beta \geq 0$ are the left-hand and right-hand spreads.

It can easily be shown that

$$
\begin{array}{r}
\widetilde{A}_{\alpha}=\left[\alpha\left(a^{L}-\left(a^{L}-\alpha\right)\right)+\left(a^{L}-\alpha\right),\right. \\
\left.-\alpha\left(\left(a^{U}+\beta\right)-a^{U}\right)+\left(a^{U}+\beta\right)\right],
\end{array}
$$

and the support of $\widetilde{A}$ is $\left(a^{L}-\alpha, a^{U}+\beta\right)$.

2.1. Arithmetic Operations. In this subsection addition, subtraction, and scalar multiplication operation of trapezoidal fuzzy numbers are reviewed $[9,15]$.

Let $\widetilde{A}=\left(a^{L}, a^{U}, \alpha, \beta\right)$ and $\widetilde{B}=\left(b^{L}, b^{U}, \theta, \gamma\right)$ be two trapezoidal fuzzy numbers; then

$$
\begin{aligned}
\widetilde{A} & =\left(a^{L}, a^{U}, \alpha, \beta\right), \\
\widetilde{B} & =\left(b^{L}, b^{U}, \gamma, \theta\right), \\
\widetilde{A}+\widetilde{B} & =\left(a^{L}+b^{L}, a^{U}+b^{U}, \alpha+\gamma, \beta+\theta\right), \\
\widetilde{A}-\widetilde{B} & =\left(a^{L}-b^{U}, a^{U}-b^{L}, \alpha+\theta, \beta+\gamma\right), \\
\lambda \widetilde{A} & = \begin{cases}\left(\lambda a^{L}, \lambda a^{U}, \lambda \alpha, \lambda \beta\right), & \lambda \geq 0, \\
\left(\lambda a^{U}, \lambda a^{L},|\lambda| \beta,|\lambda| \alpha\right), & \lambda<0 .\end{cases}
\end{aligned}
$$

\section{Interval Type 2 Fuzzy Sets}

Interval type 2 fuzzy sets (IT2FSs) play a central role in fuzzy sets as models for words and in engineering applications of type 2 fuzzy sets. These fuzzy sets are characterized by their footprints of uncertainty, which in turn are characterized by their boundaries upper and lower membership functions.

Definition 8 (see [17]). Type 2 fuzzy set $\widetilde{A}$ in the universe of discourse $X$ can be represented by type 2 membership function $\mu_{\widetilde{A}}(x, u)$ as follows:

$$
\begin{aligned}
\widetilde{A} & =\left\{\left((x, u), \mu_{\widetilde{A}}(x, u)\right) \mid \forall x \in X, \forall u \in J_{x} \subseteq[0,1], 0\right. \\
& \left.\leq \mu_{\widetilde{A}}(x, u) \leq 1\right\},
\end{aligned}
$$

where $J_{x} \subseteq[0,1]$ is the primary membership function at $x$, and

$$
\int_{u \in J_{x}} \frac{\mu_{\widetilde{A}}(x, u)}{u}
$$

indicates the second membership at $x$. For discrete situations, $\int$ is replaced by $\sum$.

Definition 9 (see $[17,18]$ ). Let $\widetilde{A}$ be type 2 fuzzy set in the universe of discourse $X$ represented by type 2 membership function $\mu_{\widetilde{A}}(x, u)$. If all $\mu_{\widetilde{A}}(x, u)=1$, then $\widetilde{A}$ is called IT2FS.
IT2FS can be regarded as a special case of type 2 fuzzy set, which is defined as

$$
\widetilde{A}=\int_{x \in X} \int_{u \in J_{x}} \frac{1}{(x, u)}=\int_{x \in X} \frac{\left[\int_{u \in J_{x}} 1 / u\right]}{x},
$$

where $x$ is the primary variable, $J_{x} \subseteq[0,1]$ is the primary membership of $x, u$ is the secondary variable, and $\int_{u \in J_{x}} 1 / u$ is the secondary membership function at $x$.

It is obvious that IT2FS $\widetilde{A}$ defined on $X$ is completely determined by the primary membership which is called the footprint of uncertainty, and the footprint of uncertainty can be expressed as follows:

$$
\operatorname{FOU}(\widetilde{A})=\bigcup_{x \in X} J_{x}=\bigcup_{x \in X}\left\{(x, u) \mid u \in J_{x} \subseteq[0,1]\right\} .
$$

Definition 10 (see $[14,19]$ ). Let $\widetilde{A}$ be IT2FS; uncertainty in the primary membership of type 2 fuzzy set consists of a bounded region called the footprint of uncertainty, which is the union of all primary membership. Footprint of uncertainty is characterized by upper membership function and lower membership function. Both of the membership functions are type 1 fuzzy sets. Upper membership function is denoted by $\bar{\mu}_{\widetilde{A}}$ and lower membership function is denoted by $\underline{\mu}_{\widetilde{A}}$, respectively.

Definition 11 (see [14]). An interval type 2 fuzzy number is called trapezoidal interval type 2 fuzzy number where the upper membership function and lower membership function are both trapezoidal fuzzy numbers; that is,

$$
\begin{aligned}
\widetilde{A}= & \left(A^{L}, A^{U}\right)=\left(\left(a_{1}^{L}, a_{2}^{L}, a_{3}^{L}, a_{4}^{L} ; H_{1}\left(A^{L}\right), H_{2}\left(A^{L}\right)\right),\right. \\
& \left.\left(a_{1}^{U}, a_{2}^{U}, a_{3}^{U}, a_{4}^{U} ; H_{1}\left(A^{U}\right), H_{2}\left(A^{U}\right)\right)\right),
\end{aligned}
$$

where $H_{j}\left(A^{L}\right)$ and $H_{j}\left(A^{U}\right),(j=1,2)$ denote membership values of the corresponding elements $a_{j+1}^{L}$ and $a_{j+1}^{U}(j=1,2)$, respectively.

Definition 12 (see [13]). The upper membership function and lower membership function of IT2FSs are type 1 membership function, respectively.

Definition 13 (see [20]). IT2FS, $\widetilde{A}$, is said to be perfectly normal if both its upper and lower membership functions are normal. It is denoted by PnIT2FS; that is,

$$
\sup \bar{\mu}_{\widetilde{A}}(x)=\sup \underline{\mu}_{\widetilde{A}}=1 .
$$

\section{Perfectly Normal IT2TrFN}

In this section, the concept of PnIT2TrFN is discussed and it is the extension work of Chiao [21] that proposed the concept of trapezoidal interval type 2 fuzzy set, in which the upper membership function and the lower membership function are represented by trapezoidal fuzzy number, which is adopted for PnIT2TrFN. PnIT2FN is a fuzzy subset of the 
real line, which is both "normal" and "convex." The operations PnIT2FSs are very complex according to the decomposition theorem [22], and the IT2FSs are usually taken in some simplified formations in applications. In subsection, the arithmetic operation on PnIT2TrFNs is formulated by proposing the extension principle.

Definition 14. Let $\widetilde{A}=\left[A^{L}, A^{U}\right]$ be PnIT2FS on $X$. Let $\widetilde{A}^{L}=$ $\left(a_{2}^{L}, a_{3}^{L}, \alpha_{L}, \beta_{L}\right)$ and $\widetilde{A}^{U}=\left(a_{2}^{U}, a_{3}^{U}, \alpha_{U}, \beta_{U}\right)$ be the lower and upper trapezoidal fuzzy number, respectively, with respect to $\widetilde{A}$ defined on the universe of discourse $X$, where $a_{2}^{L} \leq a_{3}^{L}$, $a_{2}^{U} \leq a_{3}^{U}, \alpha_{L}, \alpha_{U} \geq 0$, and $\beta_{L}, \beta_{U} \geq 0$. $\left[a_{2}^{L}, a_{3}^{L}\right]$ is the core of $\widetilde{A}^{L}$, and $\alpha_{L}, \beta_{L} \geq 0$ are the left-hand and right-hand spreads and $\left[a_{2}^{U}, a_{3}^{U}\right]$ is the core of $\widetilde{A}^{U}$, and $\alpha_{U}, \beta_{U} \geq 0$ are the lefthand and right-hand spreads. The membership functions of $x$ in $\widetilde{A}^{L}$ and $\widetilde{A}^{U}$ are expressed as follows:

$$
\begin{aligned}
& \underline{\mu}_{\widetilde{A}}(x)= \begin{cases}\frac{\left(x-a_{2}^{L}+\alpha_{L}\right)}{\alpha_{L}}, & a_{2}^{L}-\alpha_{L} \leq x \leq a_{2}^{L}, \\
1, & a_{2}^{L} \leq x \leq a_{3}^{L}, \\
-\frac{\left(x-a_{3}^{L}-\beta_{L}\right)}{\beta_{L}}, & a_{3}^{L} \leq x \leq a_{3}^{L}+\beta_{L}, \\
0, & \text { otherwise, }\end{cases} \\
& \bar{\mu}_{\widetilde{A}}(x)= \begin{cases}\frac{\left(x-a_{2}^{U}+\alpha_{U}\right)}{\alpha_{U}}, & a_{2}^{U}-\alpha_{U} \leq x \leq a_{2}^{U}, \\
1, & a_{2}^{U} \leq x \leq a_{3}^{U}, \\
-\frac{\left(x-a_{3}^{U}-\beta_{U}\right)}{\beta_{U}}, & a_{3}^{U} \leq x \leq a_{3}^{U}+\beta_{U}, \\
0, & \text { otherwise. }\end{cases}
\end{aligned}
$$

$\underline{\mu}_{\widetilde{A}}(x)$ and $\bar{\mu}_{\widetilde{A}}(x)$ are lower and upper bounds, respectively, of $\widetilde{A}$ (see Figure 1). Then, $\widetilde{A}$ is a PnIT2TrFN on $X$ and is represented by the following: $\widetilde{A}=\left[A^{L}, A^{U}\right]=\left[\left(a_{2}^{L}, a_{3}^{L}, \alpha_{L}\right.\right.$, $\left.\left.\beta_{L}\right),\left(a_{2}^{U}, a_{3}^{U}, \alpha_{U}, \beta_{U}\right)\right]$. Obviously, if $a_{2}^{L}=a_{3}^{L}$ and $a_{2}^{U}=a_{3}^{U}$ the PnIT2TrFN reduces to the perfectly normal interval type 2 triangular fuzzy number (PnIT2TFN). If $A^{L}=A^{U}$, then PnIT2TrFN $\widetilde{A}$ becomes type 1 trapezoidal fuzzy number [13, 23].

Definition 15 (primary $\alpha$-cut of PnIT2FS). The primary $\alpha$-cut of PnIT2FS is ${ }^{\alpha} \widetilde{A}=\left\{(x, u) \mid J_{x} \geq \alpha, u \in[0,1]\right\}$ which is bounded by two regions:

$$
\begin{aligned}
& { }^{\alpha} \underline{\underline{\mu}}_{\widetilde{A}}(x)=\left\{\left(x, \underline{\mu}_{\widetilde{A}}(x)\right) \mid \underline{\mu}_{\widetilde{A}}(x) \geq \alpha, \forall \alpha \in[0,1]\right\}, \\
& { }^{\alpha} \bar{\mu}_{\widetilde{A}}(x)=\left\{\left(x, \bar{\mu}_{\widetilde{A}}(x)\right) \mid \bar{\mu}_{\widetilde{A}}(x) \geq \alpha, \forall \alpha \in[0,1]\right\} .
\end{aligned}
$$

Definition 16 (crisp bounds of PnIT2FN). The crisp boundary of the primary $\alpha$-cut of PnIT2FN $\widetilde{A}=\left(A^{L}, A^{U}\right)$ is closed interval ${ }^{\alpha^{c b}} \widetilde{A}$ which will be obtained as follows: $A^{L}$ and $A^{U}$ are the lower and upper interval valued bounds of $\widetilde{A}$. Also

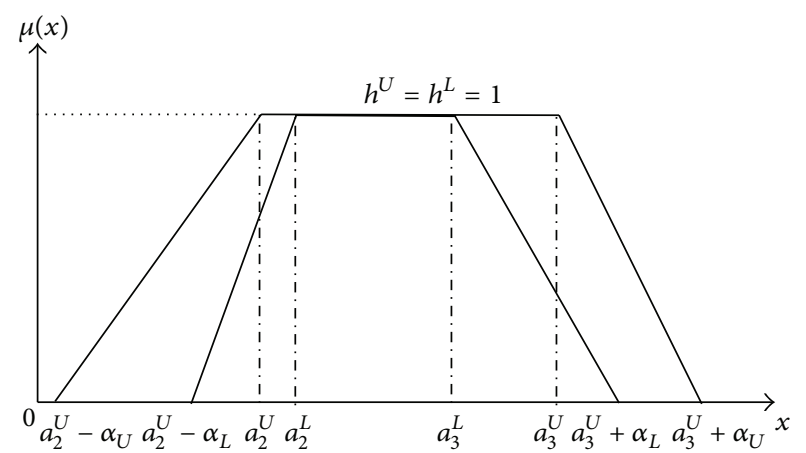

Figure 1: The lower trapezoidal membership function $\widetilde{A}^{L}$ and the upper trapezoidal membership function $\widetilde{A}^{U}$ of PnIT2FS $\widetilde{A}$.

the boundary of $A_{\alpha}^{L}$ and $A_{\alpha}^{U}$ can be defined as the boundary of the $\alpha$-cuts of each interval type 1 fuzzy set:

$$
\begin{aligned}
A_{\alpha}^{L} & =\left[\inf _{x}^{\alpha} \underline{\mu}_{\widetilde{A}}(x), \sup _{x}{ }^{\alpha} \underline{\mu}_{\widetilde{A}}(x)\right]=\left[a_{l}^{L}, a_{u}^{L}\right], \\
A_{\alpha}^{U} & =\left[\inf _{x}^{\alpha} \bar{\mu}_{\widetilde{A}}(x), \sup _{x}{ }^{\alpha} \bar{\mu}_{\widetilde{A}}(x)\right]=\left[a_{l}^{U}, a_{u}^{U}\right], \\
\alpha^{c b} \widetilde{A} & =\left[\inf _{x}\left\{{ }^{\alpha} \mu_{\widetilde{A}}(x, u)\right\}, \sup _{x}\left\{{ }^{\alpha} \mu_{\widetilde{A}}(x, u)\right\}\right] \\
& =\left[\left[a_{l}^{U}, a_{l}^{L}\right],\left[a_{u}^{L}, a_{u}^{U}\right]\right] \\
& =\left[\left[{ }^{\alpha} \bar{a}^{L},{ }^{\alpha} \underline{a}^{L}\right],\left[{ }^{\alpha} \underline{a}^{R},{ }^{\alpha} \bar{a}^{R}\right]\right]
\end{aligned}
$$

which is equivalent to, say,

$$
{ }^{c b} \mu_{\widetilde{A}} \in\left[\left[{ }^{\alpha} \bar{a}^{L},{ }^{\alpha} \underline{a}^{L}\right],\left[{ }^{\alpha} \underline{a}^{R},{ }^{\alpha} \bar{a}^{R}\right]\right] .
$$

Evidently, for PnIT2TrFN from Figure 2

$$
\alpha \bar{a}^{L} \leq{ }^{\alpha} \underline{a}^{L} \leq{ }^{\alpha} \underline{a}^{R} \leq{ }^{\alpha} \bar{a}^{R} .
$$

Definition 17. A fuzzy number $\widetilde{A}=\left(A^{L}, A^{U}\right)=\left(\left(a_{2}^{L}, a_{3}^{L}, \alpha_{L}\right.\right.$, $\left.\left.\beta_{L}\right),\left(a_{2}^{U}, a_{3}^{U}, \alpha_{U}, \beta_{U}\right)\right)$ is said to be nonnegative PnIT2TrFN if $\bar{\mu}_{\widetilde{A}}(x)=\underline{\mu}_{\widetilde{A}}=0, \forall x>0$.

Definition 18. A fuzzy number $\widetilde{A}=\left(A^{L}, A^{U}\right)=\left(\left(a_{2}^{L}, a_{3}^{L}, \alpha_{L}\right.\right.$, $\left.\left.\beta_{L}\right),\left(a_{2}^{U}, a_{3}^{U}, \alpha_{U}, \beta_{U}\right)\right)$ is said to be nonpositive PnIT2TrFN if $\underline{\mu}_{\widetilde{A}}(x)=\bar{\mu}_{\widetilde{A}}=0, \forall x<0$.

Definition 19. A fuzzy number $\widetilde{A}=\left(A^{L}, A^{U}\right)=\left(\left(a_{2}^{L}, a_{3}^{L}, \alpha_{L}\right.\right.$, $\left.\left.\beta_{L}\right),\left(a_{2}^{U}, a_{3}^{U}, \alpha_{U}, \beta_{U}\right)\right)$ is said to be zero PnIT2TrFN if $a_{2}^{L}=$ $b_{2}^{L}=0, a_{3}^{L}=b_{3}^{L}=0, \alpha_{L}=\gamma_{L}=\beta_{L}=\theta_{L}=\alpha_{U}=\gamma_{U}=0$, and $\beta_{U}=\theta_{U}=0$.

Definition 20. PnIT2TrFNs $\widetilde{A}=\left(\left(a_{2}^{L}, a_{3}^{L}, \alpha_{L}, \beta_{L}\right),\left(a_{2}^{U}, a_{3}^{U}, \alpha_{U}\right.\right.$, $\left.\left.\beta_{U}\right)\right)$ and $\widetilde{B}=\left(\left(b_{2}^{L}, b_{3}^{L}, \gamma_{L}, \theta_{L}\right),\left(b_{2}^{U}, b_{3}^{U}, \gamma_{U}, \theta_{U}\right)\right)$ are said to be identically equal to $\widetilde{A}=\widetilde{B}$ if and only if $a_{2}^{L}=b_{2}^{L}, a_{3}^{L}=b_{3}^{L}$, $\alpha_{L}=\gamma_{L}, \beta_{L}=\theta_{L}, \alpha_{U}=\gamma_{U}$, and $\beta_{U}=\theta_{U}$. 


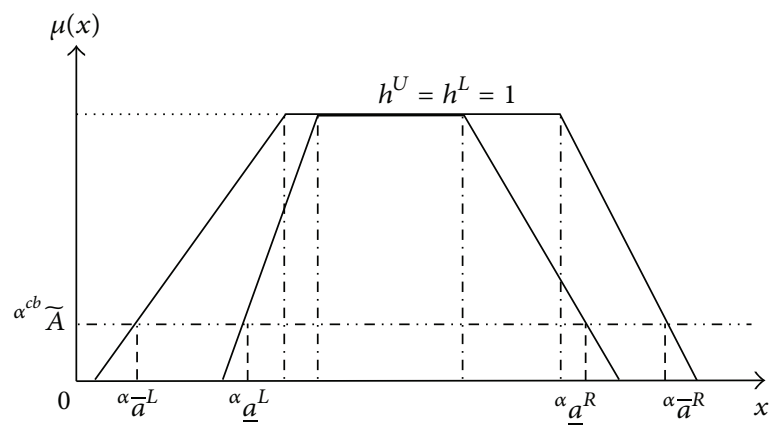

Figure 2: Crisp bounds of PnIT2TrFN.

\subsection{Arithmetic Operations on PnIT2TrFN}

Definition 21. If $\widetilde{A}=\left(\left(a_{2}^{L}, a_{3}^{L}, \alpha_{L}, \beta_{L}\right),\left(a_{2}^{U}, a_{3}^{U}, \alpha_{U}, \beta_{U}\right)\right)$ and $\widetilde{B}=\left(\left(b_{2}^{L}, b_{3}^{L}, \gamma_{L}, \theta_{L}\right),\left(b_{2}^{U}, b_{3}^{U}, \gamma_{U}, \theta_{U}\right)\right)$ are PnIT2TrFNs, then $\widetilde{C}=\widetilde{A}+\widetilde{B}$ is also PnIT2TrFN and defined by

$$
\begin{aligned}
\widetilde{C}= & \left(\left(a_{2}^{L}+b_{2}^{L}, a_{3}^{L}+b_{3}^{L}, \alpha_{L}+\gamma_{L}, \beta_{L}+\theta_{L}\right),\right. \\
& \left.\left(a_{2}^{U}+b_{2}^{U}, a_{3}^{U}+b_{3}^{U}, \alpha_{U}+\gamma_{U}, \beta_{U}+\theta_{U}\right)\right) .
\end{aligned}
$$

Definition 22. If $\widetilde{A}=\left(\left(a_{2}^{L}, a_{3}^{L}, \alpha_{L}, \beta_{L}\right),\left(a_{2}^{U}, a_{3}^{U}, \alpha_{U}, \beta_{U}\right)\right)$ and $\widetilde{B}=\left(\left(b_{2}^{L}, b_{3}^{L}, \gamma_{L}, \theta_{L}\right),\left(b_{2}^{U}, b_{3}^{U}, \gamma_{U}, \theta_{U}\right)\right)$ are PnIT2TrFNs, then $\widetilde{C}=\widetilde{A}-\widetilde{B}$ is also PnIT2TrFN and defined by

$$
\begin{gathered}
\widetilde{C}=\left(\left[a_{2}^{L}-b_{3}^{U}, a_{3}^{L}-b_{2}^{U}, \alpha_{L}+\theta_{U}, \beta_{L}+\gamma_{U}\right],\right. \\
\left.\left[a_{2}^{U}-b_{3}^{L}, a_{3}^{U}-b_{2}^{L}, \alpha_{U}+\theta_{L}, \beta_{U}+\gamma_{L}\right]\right) .
\end{gathered}
$$

Definition 23. Let $\lambda \in \mathbb{R}$. If $\widetilde{A}=\left(\left(a_{2}^{L}, a_{3}^{L}, \alpha_{L}, \beta_{L}\right),\left(a_{2}^{U}, a_{3}^{U}, \alpha_{U}\right.\right.$, $\left.\left.\beta_{U}\right)\right)$ is PnIT2TrFN, then $\widetilde{C}=\lambda \widetilde{A}$ is also PnIT2TrFN and is given by

$$
\widetilde{C}=\lambda \widetilde{A}= \begin{cases}\left(\left(\lambda a_{2}^{L}, \lambda a_{3}^{L}, \lambda \alpha_{L}, \lambda \beta_{L}\right),\left(\lambda a_{2}^{U}, \lambda a_{3}^{U}, \lambda \alpha_{U}, \lambda \beta_{U}\right)\right) ; & \text { if } \lambda \geq 0, \\ \left(\left(\lambda a_{3}^{U}, \lambda a_{2}^{U},|\lambda| \beta_{U},|\lambda| \alpha_{U}\right),\left(\lambda a_{3}^{L}, \lambda a_{2}^{L},|\lambda| \beta_{L},|\lambda| \alpha_{L}\right)\right) ; & \text { if } \lambda<0 .\end{cases}
$$

\section{The Possibility Degree of PnIT2TrFN}

Comparison of fuzzy numbers is considered one of the most important topics in fuzzy logic theory. The early and most important work in the field of comparing fuzzy numbers has been presented by Dubois and Prade [24]. On the other hand, the dominance possibility indices, which have been introduced by Negi et al., were utilized in the field of fuzzy mathematical programming $[25,26]$. The approach used in these fields was based on formulating a possibility function, whether in the case of trapezoidal fuzzy numbers or the case of triangular fuzzy numbers. In this paper, we are going to utilize the degree of possibility that the proposition stating that " $A$ is less than or equal to $B$ " is true which is proposed by Chen and Lee [13] for calculating the ranking values of perfectly normal trapezoidal interval type 2 fuzzy number. Here, the height of the upper membership function and lower membership function is considered as 1, so the modified proposition can be as follows.

Definition 24. Let $\widetilde{A}=\left(\left(a_{2}^{L}, a_{3}^{L}, \alpha_{L}, \beta_{L}\right),\left(a_{2}^{U}, a_{3}^{U}, \alpha_{U}, \beta_{U}\right)\right)$ and $\widetilde{B}=\left(\left(b_{2}^{L}, b_{3}^{L}, \gamma_{L}, \theta_{L}\right),\left(b_{2}^{U}, b_{3}^{U}, \gamma_{U}, \theta_{U}\right)\right)$ be two PnIT2TrFNs. Then the possibility degrees of lower and upper membership function are defined as follows:

$$
\begin{aligned}
& \operatorname{Pos}\left(A^{L} \leq B^{L}\right)=\max (1 \\
& -\max \left(\frac{\max \left({ }^{\alpha} \underline{a}^{L}-{ }^{\alpha} \underline{b}^{L}, 0\right)+\max \left(\left(a_{2}^{L}-b_{2}^{L}\right), 0\right)+\max \left(\left(a_{3}^{L}-b_{3}^{L}\right), 0\right)+\max \left({ }^{\alpha} \underline{a}^{R}-{ }^{\alpha} \underline{b}^{R}, 0\right)+\left({ }^{\alpha} \underline{a}^{R}-{ }^{\alpha} \underline{b}^{L}\right)}{\left|{ }^{\alpha} \underline{a}^{L}-{ }^{\alpha} \underline{b}^{L}\right|+\left|a_{2}^{L}-b_{2}^{L}\right|+\left|a_{3}^{L}-b_{3}^{L}\right|+\left|{ }^{\alpha} \underline{a}^{R}-{ }^{\alpha} \underline{b}^{R}\right|+\left({ }^{\alpha} \underline{b}^{R}-{ }^{\alpha} \underline{b}^{L}\right)+\left({ }^{\alpha} \underline{a}^{R}-{ }^{\alpha} \underline{a}^{L}\right)}, 0\right), \\
& 0)
\end{aligned}
$$




$$
\begin{aligned}
& \operatorname{Pos}\left(A^{U} \leq B^{U}\right)=\max (1 \\
& -\max \left(\frac{\max \left({ }^{\alpha} \bar{a}^{L}-{ }^{\alpha} \bar{b}^{L}, 0\right)+\max \left(\left(a_{2}^{U}-b_{2}^{U}\right), 0\right)+\max \left(\left(a_{3}^{U}-b_{3}^{U}\right), 0\right)+\max \left({ }^{\alpha} \bar{a}^{R}-{ }^{\alpha} \bar{b}^{R}, 0\right)+\left({ }^{\alpha} \bar{a}^{R}-{ }^{\alpha} \bar{b}^{L}\right)}{\left|{ }^{\alpha} \bar{a}^{L}-{ }^{\alpha} \bar{b}^{L}\right|+\left|a_{2}^{U}-b_{2}^{U}\right|+\left|a_{3}^{U}-b_{3}^{U}\right|+\left|\alpha \bar{a}^{R}-{ }^{\alpha} \bar{b}^{R}\right|+\left({ }^{\alpha} \bar{b}^{R}-\alpha{ }^{L}{ }^{L}\right)+\left({ }^{\alpha} \bar{a}^{R}-\alpha \bar{a}^{L}\right)}, 0\right), \\
& \quad \\
& \quad) .
\end{aligned}
$$

Proposition 25. Let $\widetilde{A}=\left(\left(a_{2}^{L}, a_{3}^{L}, \alpha_{L}, \beta_{L}\right),\left(a_{2}^{U}, a_{3}^{U}, \alpha_{U}, \beta_{U}\right)\right)$ and $\widetilde{B}=\left(\left(b_{2}^{L}, b_{3}^{L}, \gamma_{L}, \theta_{L}\right),\left(b_{2}^{U}, b_{3}^{U}, \gamma_{U}, \theta_{U}\right)\right)$ be two PnIT2FNs, $\operatorname{Pos}\left(A^{L} \leq B^{L}\right) \geq \alpha$, if and only if $\inf \left[A^{L}\right]_{\alpha} \leq \sup \left[B^{L}\right]_{\alpha}$; that is,

$$
\operatorname{Pos}\left(A^{L} \leq B^{L}\right) \geq \alpha \quad \text { iff }{ }^{\alpha} \underline{a}^{L} \leq{ }^{\alpha} \underline{b}^{R} .
$$

Alternatively,

$$
\operatorname{Pos}\left(A^{L} \preceq B^{L}\right)=\mu_{\operatorname{Pos}\left(A^{L}, B^{L}\right)}=\left(A^{L} \preceq{ }^{\operatorname{Pos}} B^{L}\right) .
$$

An interpretation of the $\alpha$-relation associated with possibility when comparing fuzzy numbers $A^{L}$ and $B^{L}$ is as follows. For a given level of satisfaction $\alpha \in[0,1]$, a fuzzy number $A^{L}$ is not better than $B^{L}$ with respect to fuzzy relation ${ }^{\text {Pos }}$, if the smallest value of $A^{L}$ with the degree of satisfaction (or possibility degree) being greater than or equal to $\alpha-$ is less than or equal to the largest value of $B^{L}$ with the degree of satisfaction greater than or equal to $\alpha$. Similar to the above

$$
\operatorname{Pos}\left(A^{U} \leq B^{U}\right) \geq \alpha \quad \text { iff }{ }^{\alpha} \bar{a}^{L} \leq{ }^{\alpha} \bar{b}^{R} .
$$

Proposition 26 (see $[15,23,24])$. Let $\widetilde{A}=\left(\left(a_{2}^{L}, a_{3}^{L}, \alpha_{L}, \beta_{L}\right)\right.$, $\left.\left(a_{2}^{U}, a_{3}^{U}, \alpha_{U}, \beta_{U}\right)\right)$ and $\widetilde{B}=\left(\left(b_{2}^{L}, b_{3}^{L}, \gamma_{L}, \theta_{L}\right),\left(b_{2}^{U}, b_{3}^{U}, \gamma_{U}, \theta_{U}\right)\right)$ be two PnIT2TrFNs; then

$$
\begin{aligned}
\operatorname{Pos} & \left(A^{L} \leq B^{L}\right) \\
= & \begin{cases}1, & b_{3}^{L} \geq a_{2}^{L}, \\
\frac{b_{3}^{L}-a_{2}^{L}+\theta_{L}+\alpha_{L}}{\theta_{L}+\alpha_{L}}, & 0 \leq a_{2}^{L}-b_{3}^{L} \leq \theta_{L}+\alpha_{L}, \\
0, & a_{2}^{L}-b_{3}^{L}>\theta_{L}+\alpha_{L} ;\end{cases}
\end{aligned}
$$

see Figure 3:

$$
\begin{aligned}
\operatorname{Pos} & \left(A^{U} \leq B^{U}\right) \\
& = \begin{cases}1, & b_{3}^{U} \geq a_{2}^{U}, \\
\frac{b_{3}^{U}-a_{2}^{U}+\theta_{U}+\alpha_{u}}{\theta_{U}+\alpha_{U}}, & 0 \leq a_{2}^{U}-b_{3}^{U} \leq \theta_{U}+\alpha_{U}, \\
0, & a_{2}^{U}-b_{3}^{U}>\theta_{U}+\alpha_{U} ;\end{cases}
\end{aligned}
$$

see Figure 4.
Theorem 27. Let $\widetilde{A}=\left(\left(a_{2}^{L}, a_{3}^{L}, \alpha_{L}, \beta_{L}\right),\left(a_{2}^{U}, a_{3}^{U}, \alpha_{U}, \beta_{U}\right)\right)$ and $\widetilde{B}=\left(\left(b_{2}^{L}, b_{3}^{L}, \gamma_{L}, \theta_{L}\right),\left(b_{2}^{U}, b_{3}^{U}, \gamma_{U}, \theta_{U}\right)\right)$ be two PnIT2TrFNs, $p \in$ $(0,1], \operatorname{Pos}\left(\widetilde{A}^{L} \leq \widetilde{B}^{L}\right) \geq p$, if and only if $b_{3}^{L}-a_{2}^{L} \geq(p-1)\left(\theta_{L}+\right.$ $\left.\alpha_{L}\right)$.

Proof. If $p=1$, then from $\operatorname{Pos}\left(\widetilde{A}^{L} \preceq \widetilde{B}^{L}\right) \geq 1$ one can get that $b_{3}^{L} \geq a_{2}^{L}$, and vice versa. If $0<p<1$, then $b_{3}^{L}<a_{2}^{L}$ and $b_{3}^{L}+\theta_{L}>a_{2}^{L}-\alpha_{L}, \operatorname{Pos}\left(\widetilde{A}^{L} \leq \widetilde{B}^{L}\right) \geq p$, if and only if $\left(b_{3}^{L}-a_{2}^{L}+\right.$ $\left.\theta_{L}+\alpha_{L}\right) /\left(\theta_{L}+\alpha_{L}\right) \geq p$; that is, $b_{3}^{L}-a_{2}^{L} \geq(p-1)\left(\theta_{L}+\alpha_{L}\right)$.

Theorem 28. Let $\widetilde{A}=\left(\left(a_{2}^{L}, a_{3}^{L}, \alpha_{L}, \beta_{L}\right),\left(a_{2}^{U}, a_{3}^{U}, \alpha_{U}, \beta_{U}\right)\right)$ and $\widetilde{B}=\left(\left(b_{2}^{L}, b_{3}^{L}, \gamma_{L}, \theta_{L}\right),\left(b_{2}^{U}, b_{3}^{U}, \gamma_{U}, \theta_{U}\right)\right)$ be two PnIT2TrFNs, $p \in$ $(0,1], \operatorname{Pos}\left(\widetilde{A}^{U} \preceq \widetilde{B}^{U}\right) \geq p$, if and only if $b_{3}^{U}-a_{2}^{U} \geq(p-1)\left(\theta_{U}+\right.$ $\left.\alpha_{U}\right)$.

Proof. If $p=1$, then from $\operatorname{Pos}\left(\widetilde{A}^{U} \preceq \widetilde{B}^{U}\right) \geq 1$ one can get that $b_{3}^{U} \geq a_{2}^{U}$, and vice versa. If $0<p<1$, then $b_{3}^{U}<a_{2}^{U}$ and $b_{3}^{U}+\theta_{U}>a_{2}^{U}-\alpha_{U}$, $\operatorname{Pos}\left(\widetilde{A}^{U} \leq \widetilde{B}^{U}\right) \geq p$, if and only if $\left(b_{3}^{U}-a_{2}^{U}+\theta_{U}+\alpha_{U}\right) /\left(\theta_{U}+\alpha_{U}\right) \geq p$; that is, $b_{3}^{U}-a_{2}^{U} \geq$ $(p-1)\left(\theta_{U}+\alpha_{U}\right)$

\section{Fuzzy Linear Programming}

In this section, we propose a fuzzy linear programming model with the technological coefficients and right-hand side (resources) being PnIT2FN:

$$
\begin{aligned}
\underset{x \in X}{\operatorname{Max} / \operatorname{Min}} & Z=c x \\
\text { S.t } & A x \preceq b, x \geq 0,
\end{aligned}
$$

where $A=\left(a_{i j}\right)_{m \times n}, b=\left(b_{1}, b_{2}, \ldots, b_{m}\right)^{T}, c=\left(c_{1}, c_{2}, \ldots, c_{n}\right)$, $x=\left(x_{1}, x_{2}, \ldots, x_{n}\right)^{T}$, and $a_{i j}, b_{i}$ are PnIT2FN and $x_{j}, c_{j} \in$ $\mathbb{R}(i=1,2, \ldots, m ; j=1,2, \ldots, n) . \preceq$ is type 2 fuzzy order.

Definition 29. Consider a set of right-hand side (resources) parameters of a fuzzy linear programming problem defined as PnIT2FS $\widetilde{b}$ defined on the closed interval

$$
\tilde{b}_{i} \in\left[\left[{ }^{\alpha} \bar{b}_{i}^{L},{ }^{\alpha} \underline{b}_{i}^{L}\right],\left[{ }^{\alpha} \underline{b}_{i}^{R},{ }^{\alpha} \bar{b}_{i}^{R}\right]\right] \in \mathbb{R}
$$




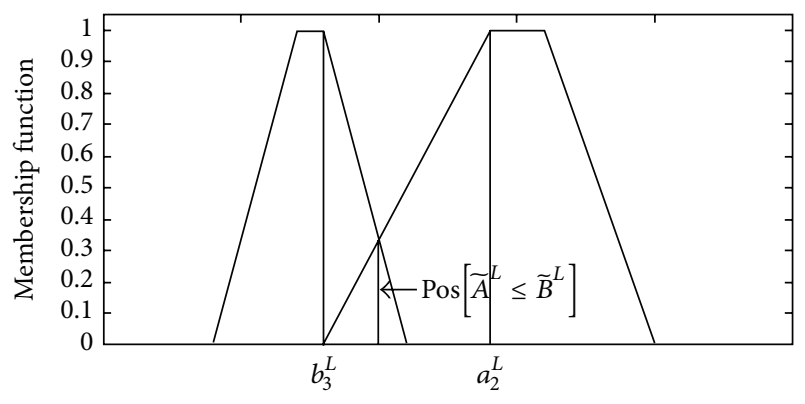

Figure 3: $\operatorname{Pos}\left[\widetilde{A}^{L} \leq \widetilde{B}^{L}\right]<1$ because $\widetilde{A}^{L}>\widetilde{B}^{L}$.

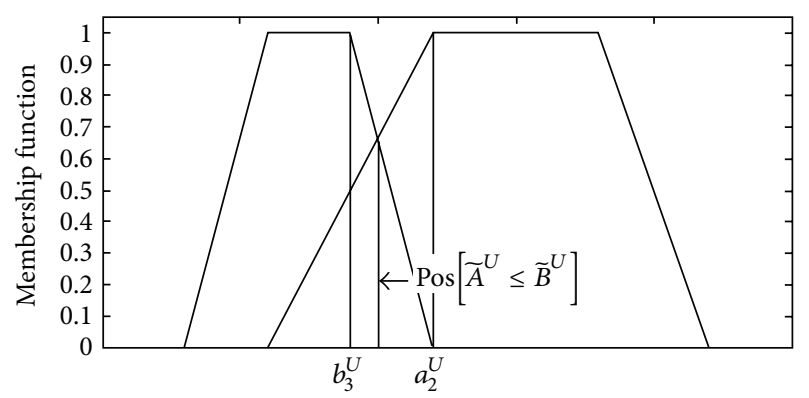

Figure 4: $\operatorname{Pos}\left[\widetilde{A}^{U} \leq \widetilde{B}^{U}\right]<1$ because $\widetilde{A}^{U}>\widetilde{B}^{U}$.

and $i \in \mathbb{N}_{n}$. The membership function which represents the fuzzy space $\operatorname{Supp}\left(\widetilde{b}_{i}\right)$ is

$$
\widetilde{b}_{i}=\int_{b_{i} \in \mathbb{R}} \frac{\left[\int_{u \in J_{b_{i}}} 1 / u\right]}{b_{i}}, \quad i \in \mathbb{N}_{n}, J_{b_{i}} \subseteq[0,1] .
$$

Here, $\widetilde{b}$ is bounded by both lower and upper primary membership function; namely,

$$
{ }^{\alpha} \underline{\mu}_{\widetilde{b}_{i}}=\left\{\left(b_{i}, u\right) \mid \underline{\mu}_{\widetilde{b}_{i}} \geq \alpha\right\},
$$

with parameters ${ }^{\alpha} \underline{b}_{i}^{L}$ and ${ }^{\alpha} \underline{b}_{i}^{R}$, and

$$
{ }^{\alpha} \bar{\mu}_{\widetilde{b}_{i}}=\left\{\left(b_{i}, u\right) \mid \bar{\mu}_{\widetilde{b}_{i}} \geq \alpha\right\}
$$

with parameters ${ }^{\alpha} \bar{b}_{i}^{L}$ and ${ }^{\alpha} \bar{b}_{i}^{R}$.

Definition 30. Consider a technological coefficient of fuzzy linear programming problem defined as PnIT2FS $\widetilde{a}_{i j}$ defined on the closed interval

$$
\begin{aligned}
\widetilde{a}_{i j} & \in\left[\inf _{a_{i j}}\left\{{ }^{\alpha} \mu_{\tilde{a}_{i j}}\left(a_{i j}, u\right)\right\}, \sup _{a_{i j}}\left\{{ }^{\alpha} \mu_{\tilde{a}_{i j}}\left(a_{i j}, u\right)\right\}\right] \\
= & {\left[\left[{ }^{\alpha} \bar{a}_{i j}^{L}, \alpha \underline{a}_{i j}^{L}\right],\left[{ }^{\alpha}{ }^{\alpha} \underline{a}_{i j}, \alpha \bar{a}_{i j}^{R}\right]\right] \in \mathbb{R}, \quad i \in \mathbb{N}_{n}, j \in \mathbb{N}_{m} . }
\end{aligned}
$$

The membership function which represents the fuzzy space $\operatorname{Supp}\left(\tilde{a}_{i j}\right)$ is

$$
\begin{aligned}
& \tilde{a}_{i j}=\int_{\tilde{a}_{i j} \in \mathbb{R}} \frac{\left[\int_{u \in \tilde{a}_{i j}} 1 / u\right]}{\widetilde{a}_{i j}}, \\
& i \in \mathbb{N}_{n}, j \in \mathbb{N}_{m}, J_{\tilde{a}_{i j}} \subseteq[0,1] .
\end{aligned}
$$

Here, $\widetilde{a}_{i j}$ is bounded by both lower and upper primary membership functions; namely

$$
{ }^{\alpha} \underline{\underline{a}}_{\widetilde{a}_{i j}}=\left\{\left(a_{i j}, u\right) \mid \underline{\mu}_{\widetilde{a}_{i j}} \geq \alpha\right\}
$$

with parameters ${ }^{\alpha} \underline{a}_{i j}^{L}$ and ${ }^{\alpha} \underline{a}_{i j}^{R}$ and

$$
{ }^{\alpha} \bar{\mu}_{\widetilde{a}_{i j}}=\left\{\left(a_{i j}, u\right) \mid \bar{\mu}_{\widetilde{a}_{i j}} \geq \alpha\right\}
$$

with parameters ${ }^{\alpha} \bar{a}_{i j}^{L}$ and ${ }^{\alpha} \bar{a}_{i j}^{R}$.

Proposition 31. Let $\tilde{a}_{i j} \in F(\mathbb{R}), x_{j} \geq 0, i \in \mathbb{N}_{n}, j \in \mathbb{N}_{m}$. Then the fuzzy set $\sum_{j=1}^{n} \widetilde{a}_{i j} x_{j}$ defined by the extension principle is again a fuzzy number.

Let $\widetilde{P}=\preceq^{\text {Pos }}$ be a fuzzy relation [27] fuzzy extension of the usual binary relation $\leq$ on $\mathbb{R}$. The fuzzy linear programming problem associated with a standard linear programming problem is denoted as

$$
\begin{aligned}
\operatorname{Max} / \operatorname{Min} & Z=\sum_{j=1}^{n} c_{j} x_{j} \\
\text { S.t } & \left(\sum_{j=1}^{n} \widetilde{a}_{i j} x_{j}\right) \widetilde{P} \widetilde{b}_{i}, \quad i=1,2, \ldots, m \\
& x_{j} \geq 0, \quad j=1,2, \ldots, n .
\end{aligned}
$$

In (36) the value $\sum_{j=1}^{n} \widetilde{a}_{i j} x_{j} \in F(\mathbb{R})$ is compared with a fuzzy number $\widetilde{b}_{i} \in F(\mathbb{R})$ by sum fuzzy relation. The maximization of the objective function is denoted by $\operatorname{Max} Z=\sum_{j=1}^{n} c_{j} x_{j}$.

Definition 32. Let $\mu_{\widetilde{a}_{i j}}: \mathbb{R} \rightarrow[0,1]$ and $\mu_{\tilde{b}_{i}}: \mathbb{R} \rightarrow[0,1]$, $i \in \mathbb{N}_{n}, j \in \mathbb{N}_{m}$, be membership function of fuzzy numbers $\widetilde{a}_{i j}$ and $\widetilde{b}_{i}$, respectively. Let $\widetilde{P}$ be a fuzzy extension of a binary relation $\leq$ on $\mathbb{R}$. A fuzzy set $\widetilde{X}$, whose membership function $\mu_{\widetilde{X}}$ is defined as $x \in \mathbb{R}^{n}$ by

$$
\mu_{\widetilde{X}}= \begin{cases}\min \left\{\mu_{\widetilde{P}}\left(\widetilde{a}_{11} x_{1}+\cdots+\widetilde{a}_{1 n} x_{n}, \widetilde{b}_{1}\right), \ldots, \mu_{\widetilde{P}}\left(\widetilde{a}_{m 1} x_{1}+\cdots+\widetilde{a}_{m n} x_{n}, \widetilde{b}_{m}\right)\right\}, & \text { if } x_{j} \geq 0, j=1,2, \ldots, n, \\ 0, & \text { otherwise, }\end{cases}
$$


is called the fuzzy set of feasible regions of the fuzzy linear programming problem (36). For $\alpha \in(0,1]$, a vector $x \in$ $[\widetilde{X}]_{\alpha}$ is called the $\alpha$-feasible solution of the fuzzy linear programming problem.

Notice that the feasible region $\widetilde{X}$ of the fuzzy linear programming problem is a fuzzy set. On the other hand, $\alpha$ feasible solution is a vector belonging to the $\alpha$-cut of the feasible region $\widetilde{X}$. If all the coefficients $\widetilde{a}_{i j}$ and $\widetilde{b}_{i}$ are crisp fuzzy number, that is, they are equivalent to the corresponding crisp fuzzy number, then the fuzzy feasible region is equivalent to the set of all feasible solutions of the corresponding classical linear programming.

6.1. Type 2 Fuzzy Linear Programming Problem. Let us consider the following fuzzy linear programming problem with right-hand side (resources) and technology coefficients are PNIT2TrFNs:

$$
\begin{aligned}
\operatorname{Max} / \operatorname{Min} & Z=\sum_{j=1}^{n} c_{j} x_{j} \\
\text { S.t } & \sum_{j=1}^{n} \widetilde{a}_{i j} x_{j} \leq \widetilde{b}_{i}, \quad i=1,2, \ldots, m \\
& x_{j} \geq 0, \quad j=1,2, \ldots, n,
\end{aligned}
$$

where $\widetilde{a}_{i j}=\left(\left(\widetilde{a}_{i j}\right)^{L},\left(\widetilde{a}_{i j}\right)^{U}\right)=\left(\left(\left(a_{i j}\right)_{2}^{L},\left(a_{i j}\right)_{3}^{L},\left(\alpha_{i j}\right)_{L},\left(\beta_{i j}\right)_{L}\right)\right.$, $\left.\left(\left(a_{i j}\right)_{2}^{U},\left(a_{i j}\right)_{3}^{U},\left(\alpha_{i j}\right)_{U},\left(\beta_{i j}\right)_{U}\right)\right), \widetilde{b}_{i}=\left(\left(\widetilde{b}_{i}\right)^{L},\left(\widetilde{b}_{i}\right)^{U}\right)=\left(\left(\left(b_{i}\right)_{2}^{L}\right.\right.$, $\left.\left.\left(b_{i}\right)_{3}^{L},\left(\gamma_{i}\right)_{L},\left(\theta_{i}\right)_{L}\right),\left(\left(b_{i}\right)_{2}^{U},\left(b_{i}\right)_{3}^{U},\left(\gamma_{i}\right)_{U},\left(\theta_{i}\right)_{U}\right)\right)$ are PnIT2TrFNs, $c_{i}$ are crisp coefficients of the objective, and $x_{i}$ are the decision variable.

From Definitions 15, 21, 22, and 23 and (38),

$$
\begin{aligned}
& \text { Max/Min } Z=\sum_{j=1}^{n} c_{j} x_{j} \\
& \text { S.t } \sum_{j=1}^{n} \widetilde{a}_{i j}^{L} x_{j} \leq \widetilde{b}_{i}^{L}, \quad i=1,2, \ldots, m \\
& x_{j} \geq 0, \quad j=1,2, \ldots, n, \\
& \text { Max/Min } \quad Z=\sum_{j=1}^{n} c_{j} x_{j} \\
& \text { S.t } \quad \sum_{j=1}^{n} \widetilde{a}_{i j}^{U} x_{j} \leq \widetilde{b}_{i}^{U}, \quad i=1,2, \ldots, m \\
& x_{j} \geq 0, \quad j=1,2, \ldots, n .
\end{aligned}
$$

Based on the relation of possibility we can rewrite (39) as follows:

$$
\begin{aligned}
\operatorname{Max} / \operatorname{Min} & Z=\sum_{j=1}^{n} c_{j} x_{j} \\
\text { S.t } & \tilde{a}_{i 1}^{L} x_{1}+\tilde{a}_{i 2}^{L} x_{2}+\cdots+\tilde{a}_{i n}^{L} x_{n} \leq{ }^{\operatorname{Pos}} \tilde{b}_{i}^{L}, \\
& \quad i=1,2, \ldots, m, \\
& x_{j} \geq 0, \quad j=1,2, \ldots, n,
\end{aligned}
$$

$$
\begin{aligned}
\operatorname{Max} / \operatorname{Min} \quad & Z=\sum_{j=1}^{n} c_{j} x_{j} \\
\text { S.t } \quad & \tilde{a}_{i 1}^{U} x_{1}+\tilde{a}_{i 2}^{U} x_{2}+\cdots+\tilde{a}_{i n}^{U} x_{n} \leq{ }^{\text {Pos } \tilde{b}_{i}^{U},} \\
& \quad i=1,2, \ldots, m, \\
& x_{j} \geq 0, \quad j=1,2, \ldots, n .
\end{aligned}
$$

With respect to constraints of linear programming possibility, the optimal solution is completely determined by degrees of possibility. So, we can obtain the optimal solution of linear programming possibility at $p$-cut levels by solving the two crisp linear programming problems using Barnes algorithm:

$$
\begin{aligned}
\operatorname{Max} / \operatorname{Min} \quad & Z=\sum_{j=1}^{n} c_{j} x_{j} \\
\text { S.t } \quad & \operatorname{Pos}\left(\tilde{a}_{i 1}^{L} x_{1}+\tilde{a}_{i 2}^{L} x_{2}+\cdots+\tilde{a}_{i n}^{L} x_{n} \leq \widetilde{b}_{i}^{L}\right) \\
& \geq p, \quad i=1,2, \ldots, m, \\
& x_{j} \geq 0, \quad j=1,2, \ldots, n, \quad \\
\operatorname{Max} / \operatorname{Min} \quad & Z=\sum_{j=1}^{n} c_{j} x_{j} \\
\text { S.t } \quad & \operatorname{Pos}\left(\widetilde{a}_{i 1}^{U} x_{1}+\widetilde{a}_{i 2}^{U} x_{2}+\cdots+\tilde{a}_{i n}^{U} x_{n} \leq \widetilde{b}_{i}^{U}\right) \\
& \geq p, \quad i=1,2, \ldots, m, \\
& x_{j} \geq 0, \quad j=1,2, \ldots, n .
\end{aligned}
$$

From Theorems 27 and 28 we have

$$
\begin{aligned}
\underline{\left(L P P_{p}\right)}=\operatorname{Max} / \operatorname{Min} \quad Z=\sum_{j=1}^{n} c_{j} x_{j} \\
\text { S.t } \quad \underline{b}^{U}-\underline{A}^{L} x \geq(p-1)(\underline{\alpha} x+\underline{\theta}), \\
x_{j} \geq 0, j=1,2, \ldots, n, \\
\overline{\left(L P P_{p}\right)}=\operatorname{Max} / \operatorname{Min} \quad Z=\sum_{j=1}^{n} c_{j} x_{j} \\
\text { S.t } \quad \bar{b}^{U}-\bar{A}^{L} x \geq(p-1)(\bar{\alpha} x+\bar{\theta}), \\
x_{j} \geq 0, j=1,2, \ldots, n,
\end{aligned}
$$

where $\underline{A}^{L}=\left\{\left(a_{i j}\right)_{2}^{L}\right\}, \bar{A}^{L}=\left\{\left(a_{i j}\right)_{2}^{U}\right\}, \underline{b}^{U}=\left\{\left(b_{i}\right)_{3}^{L}\right\}, \bar{b}^{U}=\left\{\left(b_{i}\right)_{3}^{U}\right\}$, $\underline{\alpha}=\left\{\left(\alpha_{i j}\right)_{L}\right\}, \bar{\alpha}=\left\{\left(\alpha_{i j}\right)_{U}\right\}, \underline{\beta}=\left\{\left(\beta_{i j}\right)_{L}\right\}, \bar{\beta}=\left\{\left(\beta_{i j}\right)_{U}\right\}, \underline{\gamma}=$ $\left\{\left(\gamma_{i}\right)_{L}\right\}, \bar{\gamma}=\left\{\left(\gamma_{i}\right)_{U}\right\}, \underline{\theta}=\left\{\left(\bar{\theta}_{i}\right)_{L}\right\}, \bar{\theta}=\left\{\left(\theta_{i}\right)_{U}\right\}$, and $x=x_{j}$, $j=1,2, \ldots, n, i=1,2, \ldots, m$. 
The feasible regions of $\left(L P P_{p}\right)$ and $\overline{\left(L P P_{p}\right)}$ are denoted by

$$
\begin{aligned}
& \underline{X}_{p}^{P}=\left\{x \in R^{n} \mid \underline{b}^{U}-\underline{A}^{L} x \geq(p-1)(\underline{\alpha} x+\underline{\theta})\right\}, \\
& \bar{X}_{p}^{P}=\left\{x \in R^{n} \mid \bar{b}^{U}-\bar{A}^{L} x \geq(p-1)(\bar{\alpha} x+\bar{\theta})\right\} .
\end{aligned}
$$

Assume the optimal solutions of $\left(L P P_{p}\right)$ and $\overline{\left(L P P_{p}\right)}$ are $\underline{Z}_{p}^{P}$ and $\bar{Z}_{p}^{P}$, respectively.

Theorem 33. For linear programming $\left(L P P_{p}\right)$ and programming $\overline{\left(L P P_{p}\right)}, 0 \leq p_{1} \leq p_{2} \leq 1$, and then $\underline{X}_{p_{2}}^{P} \subseteq \underline{X}_{p_{1}}^{P}, \bar{X}_{p_{2}}^{P} \subseteq$ $\bar{X}_{p_{1}}^{P}$ and $\underline{Z}_{p_{1}}^{P} \geq \underline{Z}_{p_{2}}^{P}, \bar{Z}_{p_{1}}^{P} \geq \bar{Z}_{p_{2}}^{P}$.

Proof. If $x \in \underline{X}_{p_{2}}^{P}$, we have $\underline{b}^{U}-\underline{A}^{L} x \geq\left(p_{2}-1\right)(\underline{\alpha} x+\underline{\theta})$, from $\beta>0, \gamma>0$, and $x>0$; then $\underline{b}^{U}-\underline{A}^{L} x \geq\left(p_{2}-1\right)(\underline{\alpha} x+\underline{\theta}) \geq$ $\left(p_{1}-1\right)(\underline{\alpha} x+\underline{\theta})$, so $x \in \underline{X}_{p_{1}}^{P}$; that is, $\underline{X}_{p_{2}}^{P} \subseteq \underline{X}_{p_{1}}^{P}$ and $\underline{Z}_{p_{1}}^{P} \geq \underline{Z}_{p_{2}}^{P}$. In a similar way $\bar{X}_{p_{2}}^{P} \subseteq \bar{X}_{p_{1}}^{P}$ and $\bar{Z}_{p_{1}}^{P} \geq \bar{Z}_{p_{2}}^{P}$.

Theorem 34. For linear programming $\left(L P P_{p}\right)$ and programming $\overline{\left(L P P_{p}\right)}, 0 \leq p_{1} \leq p_{2} \leq 1$, and then $\underline{X}_{p_{2}}^{P} \subseteq \bar{X}_{p_{1}}^{P}$ and $\underline{Z}_{p_{1}}^{P} \geq \bar{Z}_{p_{2}}^{P}$.

Proof. If $x \in \underline{X}_{p_{2}}^{P}$, we have $\underline{b}^{U}-\underline{A}^{L} x \geq\left(p_{2}-1\right)(\underline{\alpha} x+\underline{\theta})$, from $\underline{\beta}>0, \underline{\gamma}>0$, and $x>0$; then $\underline{b}^{U}-\underline{A}^{L} x \geq\left(p_{2}-1\right)(\underline{\alpha} x+$ $\underline{\theta}) \geq\left(p_{1}-1\right)(\bar{\alpha} x+\bar{\theta})$, so $x \in \bar{X}_{p_{1}}^{P}$; that is, $\underline{X}_{p_{2}}^{P} \subseteq \bar{X}_{p_{1}}^{P}$ and $\bar{Z}_{p_{1}}^{P} \geq \underline{Z}_{p_{2}}^{P}$.

From Theorems 33 and 34, we obtain that $\underline{Z}_{p}^{P}, \bar{Z}_{p}^{P}$ constitute the lower and upper bound of fuzzy objective value at level $p$. Then the objective value of $Z_{p}^{P}$ at level $p$ is the mean of $\left[\underline{Z}_{p}^{P}, \bar{Z}_{p}^{P}\right]$.

\section{Numerical Illustration}

An application of the proposed method is introduced with an example, where all its technological coefficients and righthand side (resources) are defined as PnIT2FNs. The optimal solution will be obtained in terms of crisp for the following problem.

The company plans to manufacture two types of products. The selling prices for these products are as follows: $P_{1}$ costs Rs. 1,750 per unit and $P_{2}$ costs Rs. 2,000 per unit. Daily production volume of each type of these products is constrained by available man hours and available machine hours. The production specifications for the given problem situation are presented in as shown in Table 1:

$$
\begin{array}{ll}
\operatorname{Max} & Z=c_{1} x_{1}+c_{2} x_{2} \\
\text { S.t } & \tilde{a}_{11} x_{1}+\tilde{a}_{12} x_{2} \preceq \tilde{b}_{1}
\end{array}
$$

TABLE 1

\begin{tabular}{lccc}
\hline \multicolumn{4}{c}{ Resource requirement/unit } \\
Resource & $P_{1}$ & $P_{2}$ & Availability \\
\hline Man hours (in minutes) & Around 4 & Around 8 & Around 32 \\
Machine hours (in minutes) & Around 6 & Around 4 & Around 36 \\
\hline
\end{tabular}

$$
\begin{aligned}
& \tilde{a}_{21} x_{1}+\tilde{a}_{22} x_{2} \leq \widetilde{b}_{2} \\
& x_{j} \geq 0, \quad j=1,2,
\end{aligned}
$$

where $\widetilde{a}_{11}=[(230,250,20,10),(230,250,30,20)], \widetilde{a}_{12}=$ $[(460,500,40,20),(460,500,60,40)], \tilde{a}_{21}=[(345,375,30$, 15), $(345,375,45,30)], \widetilde{a}_{22}=[(230,250,20,10),(230,250$, $30,20)], \widetilde{b}_{1}=[(1840,2000,160,80),(1840,2000,240,160)]$, $\widetilde{b}_{2}=[(2070,2250,180,90),(2070,2250,270,180)], c_{1}=$ 1750 , and $c_{2}=2000$. From (42) we have the following crisp optimal problem based on the level $p$ :

$$
\begin{aligned}
\operatorname{Max} & \underline{Z}_{p}^{P}=1750 x_{1}+2000 x_{2} \\
\text { S.t } & (210+20 p) x_{1}+(420+40 p) x_{2} \\
& \leq 2080-80 p \\
& (315+30 p) x_{1}+(210+20 p) x_{2} \\
& \leq 2340-90 p \\
& x_{1}, x_{2} \geq 0 \\
\operatorname{Max} & \bar{Z}_{p}^{P}=1750 x_{1}+2000 x_{2} \\
\text { S.t } & (200+30 p) x_{1}+(400+60 p) x_{2} \\
& \leq 2160-160 p \\
& (300+45 p) x_{1}+(200+30 p) x_{2} \\
& \leq 2430-180 p \\
& x_{1}, x_{2} \geq 0 .
\end{aligned}
$$

For different cut level $p$, we can get different optimal solution and denote by $\underline{Z}_{p}^{P}, \bar{Z}_{p}^{P}$ the optimal solution (see Figure 5 and Table 2) [28] of the crisp programming (45) and programming (46), respectively.

\section{Conclusion}

In this paper a method to do perfectly normal interval type 2 fuzzy arithmetic operation using extension principle is presented and defined the possibility degrees of upper and lower membership function to compare perfectly normal interval type 2 fuzzy numbers. These functions are defined based on the strength of upper and lower membership function of perfectly normal interval type 2 fuzzy numbers. Meanwhile, some properties and theorems were proved. Then, the lower and upper fuzzy satisfaction based on possibility degrees of 
TABLE 2: Optimal solution at different possibility levels.

\begin{tabular}{lcccccccccc}
\hline$p$-cut & 0.1 & 0.2 & 0.3 & 0.4 & 0.5 & 0.6 & 0.7 & 0.8 & 0.9 & 1 \\
\hline$\underline{Z}_{p}^{P}$ & 14354.9528 & 14165.8878 & 13980.324 & 13798.1651 & 13619.3181 & 13443.6937 & 13271.2053 & 13101.7699 & 12935.3069 & 12771.7393 \\
$\bar{Z}_{p}^{P}$ & 15512.3153 & 15172.3301 & 14842.1051 & 14521.2264 & 14209.3022 & 13905.9633 & 13610.8597 & 13323.6607 & 13044.0529 & 12771.7393 \\
$Z_{p}^{P}$ & 14933.6341 & 14669.1090 & 14411.2146 & 14159.6958 & 13914.3102 & 13674.8285 & 13441.0325 & 13212.7153 & 12989.6799 & 12771.7393 \\
\hline
\end{tabular}

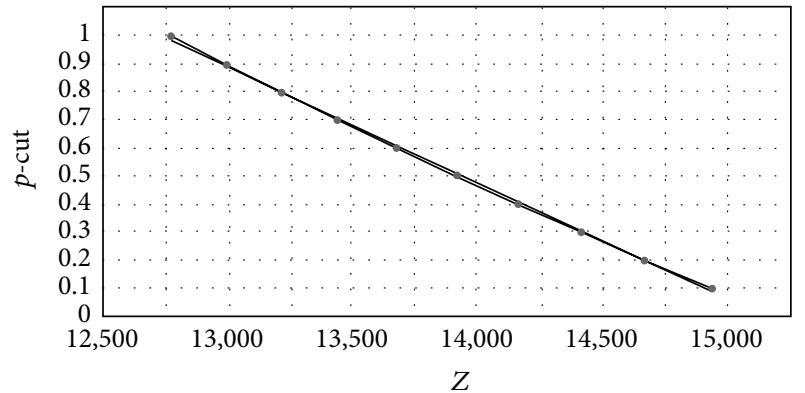

FIGURE 5: Optimal solution at different possibility levels.

membership function, respectively, are defined. These fuzzy satisfactions create two nonstrict order relations on the set of overlapping intervals. Also, linear programming problem was introduced with resources and technology coefficients of perfectly normal interval type 2 fuzzy numbers. Then, the possibility degrees of membership function were applied in order to interpret inequality constraints with interval coefficients. According to the definition of possibility degrees of membership function and their properties, the inequality constraints with interval coefficients were reduced in their satisfactory crisp equivalent forms. Finally, the decision maker can get the crisp optimal solution of the problem for every grade $\alpha \in[0,1]$ which was obtained by using Barnes algorithm and then mean value is selected according to the decision maker optimistic attitude.

\section{Conflict of Interests}

The authors declare that there is no conflict of interests regarding the publication of this paper.

\section{References}

[1] L. A. Zadeh, "Fuzzy sets," Information and Computation, vol. 8, pp. 338-353, 1965.

[2] H. Tanaka, T. Okuda, and K. Asai, "On fuzzy-mathematical programming," Journal of Cybernetics, vol. 3, no. 4, pp. 37-46, 1973.

[3] R. E. Bellman and L. A. Zadeh, "Decision-making in a fuzzy environment," Management Science, vol. 17, pp. B141-B164, 1970/71.

[4] H.-J. Zimmermann, "Fuzzy programming and linear programming with several objective functions," Fuzzy Sets and Systems, vol. 1, no. 1, pp. 45-55, 1978.

[5] S. Deng, L. Zhou, and X. Wang, "Solving the fuzzy bilevel linear programming with multiple followers through structured element method," Mathematical Problems in Engineering, vol. 2014, Article ID 418594, 6 pages, 2014.

[6] Y.-H. Zhong, Y.-L. Jia, D. Chen, and Y. Yang, "Interior point method for solving fuzzy number linear programming problems using linear ranking function," Journal of Applied Mathematics, vol. 2013, Article ID 795098, 9 pages, 2013.

[7] H. Cheng, W. Huang, and J. Cai, "Solving a fully fuzzy linear programming problem through compromise programming," Journal of Applied Mathematics, vol. 2013, Article ID 726296, 10 pages, 2013.

[8] D. Chakraborty, D. K. Jana, and T. K. Royl, "A new approach to solve intuitionistic fuzzy optimization problem using possibility, necessity, and credibility measures," International Journal of Engineering Mathematics, vol. 2014, Article ID 593185, 12 pages, 2014.

[9] H. Le and Z. Gogne, "Fuzzy linear programming with possibility and necessity relation," in Fuzzy Information and Engineering 2010, vol. 78 of Advances in Intelligent and Soft Computing, pp. 305-311, Springer, Berlin, Germany, 2010.

[10] J. C. Figueroa-García and G. Hernández, "A method for solving linear programming models with interval type-2 fuzzy constraints," Pesquisa Operacional, vol. 34, no. 1, pp. 73-89, 2014.

[11] J. C. F. García, "A general model for linear programming with interval type-2 fuzzy technological coefficients," in Proceedings of the IEEE Annual Meeting of the North American Fuzzy Information Processing Society (NAFIPS '12), pp. 1-4, Berkeley, Calif, USA, August 2012.

[12] J. C. F. García, "Linear programming with interval type-2 fuzzy right hand side parameters," in Proceedings of the Annual Meeting of the North American Fuzzy Information Processing Society (NAFIPS '08), pp. 1-6, IEEE, New York, NY, USA, May 2008.

[13] S.-M. Chen and L.-W. Lee, "Fuzzy multiple attributes group decision-making based on the ranking values and the arithmetic operations of interval type- 2 fuzzy sets," Expert Systems with Applications, vol. 37, no. 1, pp. 824-833, 2010.

[14] J. Hu, Y. Zhang, X. Chen, and Y. Liu, "Multi-criteria decision making method based on possibility degree of interval type2 fuzzy number," Knowledge-Based Systems, vol. 43, pp. 21-29, 2013.

[15] R. Fuller, Fuzzy Reasoning and Fuzzy Optimization, Turku Centre for Computer Science, 1998.

[16] H.-C. Wu, "Duality theory in fuzzy linear programming problems with fuzzy coefficients," Fuzzy Optimization and Decision Making, vol. 2, no. 1, pp. 61-73, 2003.

[17] J. Qin and X. Liu, "Frank aggregation operators for triangular interval type-2 fuzzy set and its application in multiple attribute group decision making," Journal of Applied Mathematics, vol. 2014, Article ID 923213, 24 pages, 2014.

[18] Z. M. Zhang and S. H. Zhang, "A novel approach to multi attribute group decision making based on trapezoidal interval type-2 fuzzy soft sets," Applied Mathematical Modelling, vol. 37, no. 7, pp. 4948-4971, 2013. 
[19] Y. Gong, "Fuzzy multi-attribute group decision making method based on interval type-2 fuzzy sets and applications to global supplier selection," International Journal of Fuzzy Systems, vol. 15, no. 4, pp. 392-400, 2013.

[20] D. S. Dinagar and A. Anbalagan, "A new type-2 fuzzy number arithmetic using extension principle," in Proceedings of the 1st International Conference on Advances in Engineering, Science and Management (ICAESM '12), pp. 113-118, March 2012.

[21] K.-P. Chiao, "Multiple criteria group decision making with triangular interval type-2 fuzzy sets," in Proceedings of the IEEE International Conference on Fuzzy Systems (FUZZ '11), pp. 25752582, IEEE, Taipei, Taiwan, June 2011.

[22] J. M. Mendel, R. I. John, and F. Liu, "Interval type-2 fuzzy logic systems made simple," IEEE Transactions on Fuzzy Systems, vol. 14, no. 6, pp. 808-821, 2006.

[23] X. Liu, "Measuring the satisfaction of constraints in fuzzy linear programming," Fuzzy Sets and Systems, vol. 122, no. 2, pp. 263275, 2001.

[24] D. Dubois and H. Prade, "Ranking fuzzy numbers in the setting of possibility theory," Information Sciences, vol. 30, no. 3, pp. 183-224, 1983.

[25] D. S. Negi and E. S. Lee, "Possibility programming by the comparison of fuzzy numbers," Computers \& Mathematics with Applications, vol. 25, no. 9, pp. 43-50, 1993.

[26] M. G. Iskander, "Comparison of fuzzy numbers using possibility programming: comments and new concepts," Computers \& Mathematics with Applications, vol. 43, no. 6-7, pp. 833-840, 2002.

[27] J. Ramik, "Duality in fuzzy linear programming with possibility and necessity relations," Fuzzy Sets and Systems, vol. 157, no. 10, pp. 1283-1302, 2006.

[28] G. R. Lindfield and J. E. T. Penny, Numerical methods Using Matlab, Academic Press, 3rd edition, 2013. 


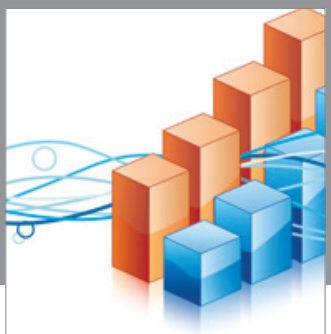

Advances in

Operations Research

vatem alat4

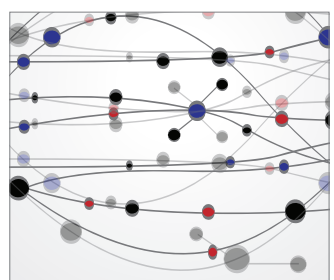

\section{The Scientific} World Journal
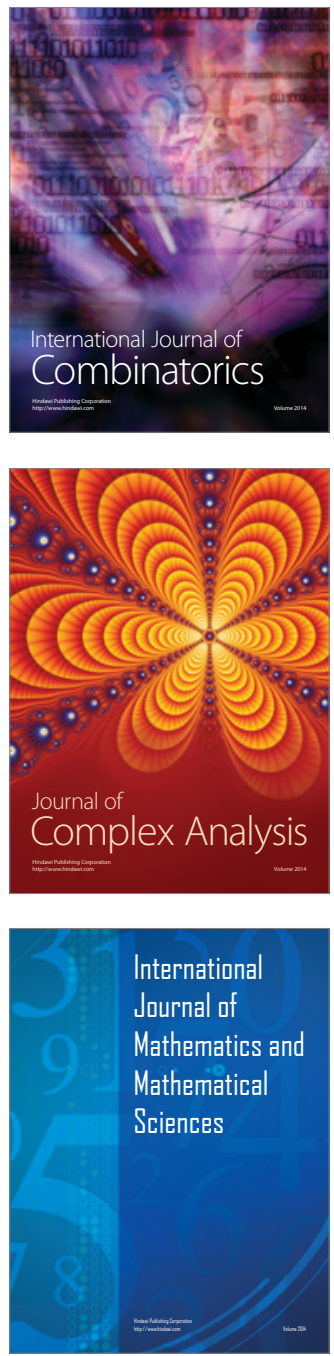
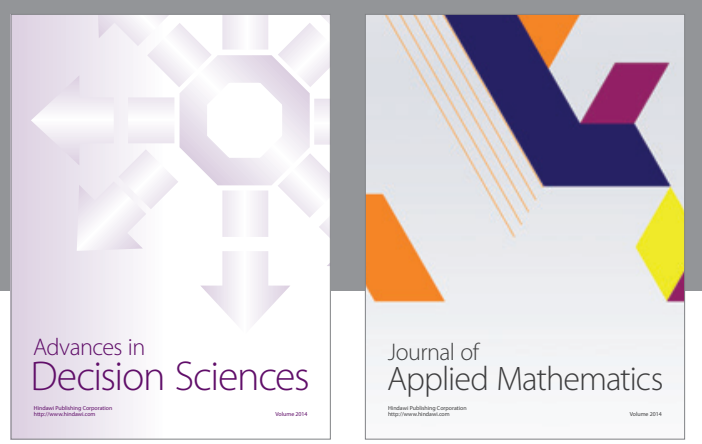

Algebra

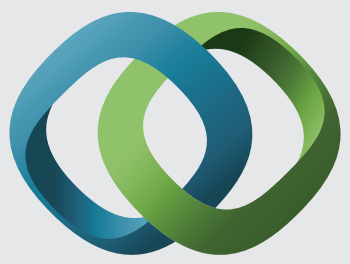

\section{Hindawi}

Submit your manuscripts at

http://www.hindawi.com
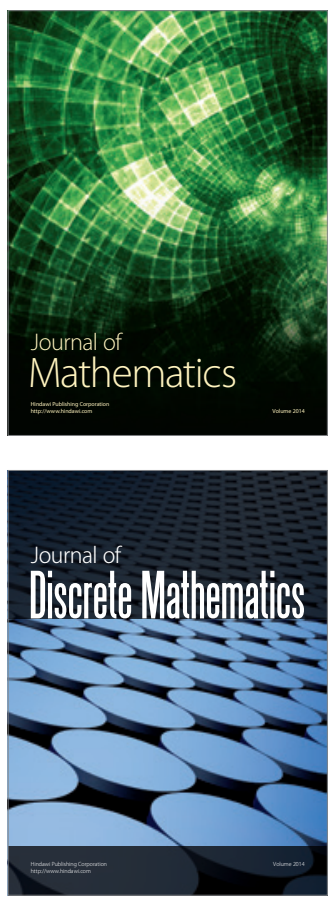

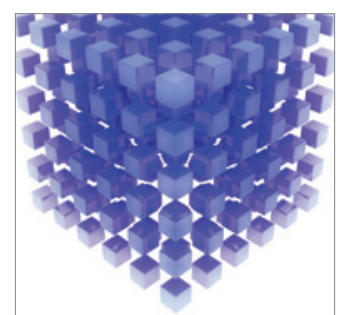

Mathematical Problems in Engineering
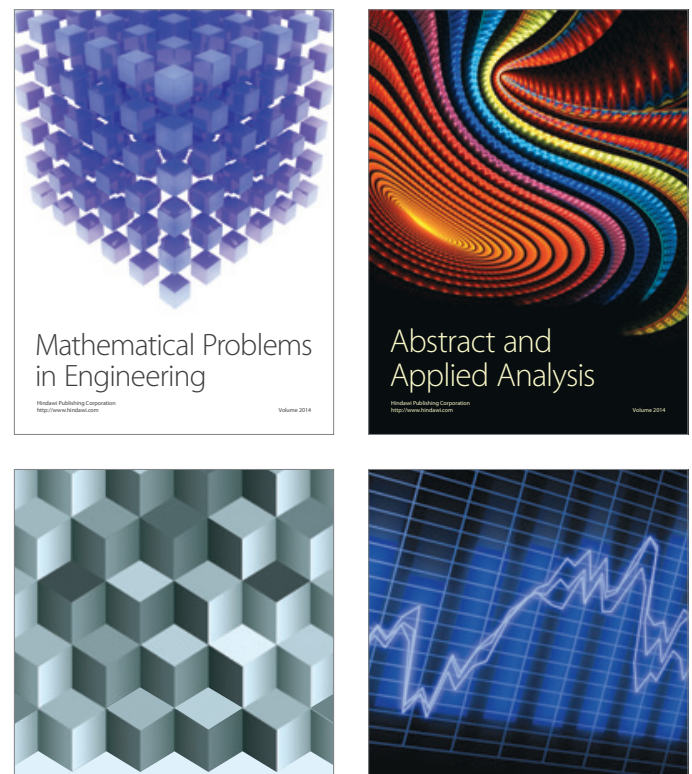

Journal of

Function Spaces

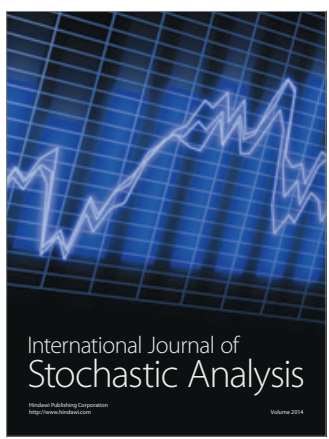

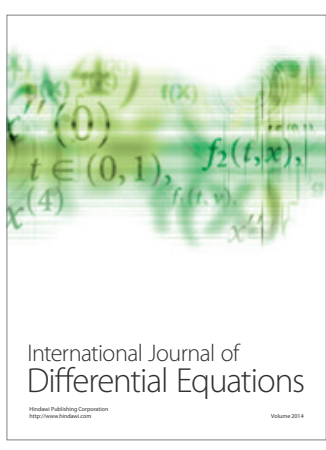
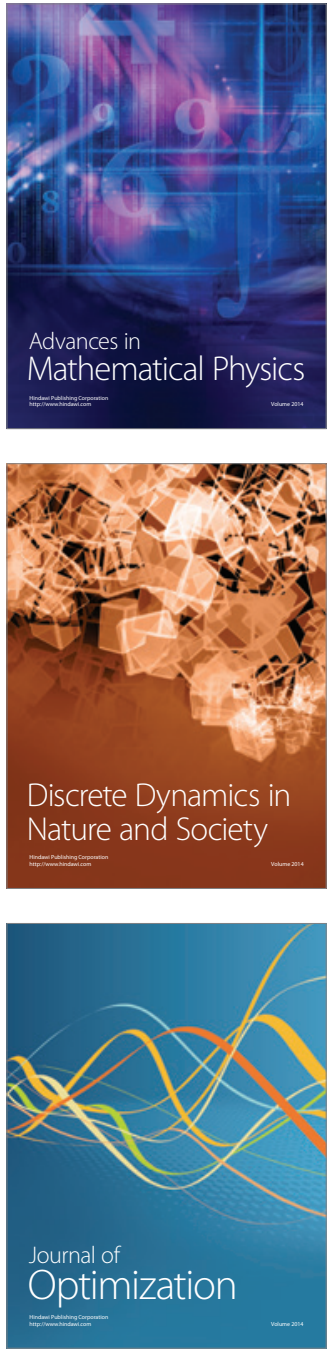